\title{
Capacidad antioxidante y metabolitos bioactivos in vitro del aceite esencial de Tagetes erecta $y$ Tagetes patula
}

Antioxidant capacity and bioactive metabolites in vitro of the essential oil of Tagetes erecta and Tagetes patula

Capacidade antioxidante e metabólitos bioativos in vitro do óleo essencial de Tagetes erecta e Tagetes patula

\section{ARTÍCULO GENERAL}

Peter Llimpe Perez

peter.1limpe@unh.edu.pe

hitps:/orcid org 0000-0003-2422-6250

Universidad Nacional de

Huancavelica, Huancavelica Perú
Lissete Lourdes Aguirre Huayhua

lissete.aguirre@unh.edu.pe

https $/ /$ orcid org/0000-0003-2450-5153

Universidad Nacional de

Huancavelica, Huancavelica Perú

Oliver Taype Landeo
oliver.taipe@unh.edu.pe
hittps://orcid.org0000-0003-4197-1164
Universidad Nacional de
Huancavelica, Huancavelica -
Perú

Oliver Taype Landeo

ps://orcid org/0000-0003-4197-1164

Universidad Nacional de Perú
Franklin Ore Areche

frankin.ore@unh.edu.pe

https:/ orcid org/0000-0002-7168-1742

Universidad Nacional de

Huancavelica, Huancavelica Perú

Recibido 08 de Noviembre 2020 | Arbitrado y aceptado 28 de Noviembre 2020 | Publicado en 05 Marzo 2021

\section{RESUMEN}

La investigación turo el objetivo de investigar los metabolitos bioactivos y actividad antioxidante de aceites esenciales de dos especies del género Tagetes. El aceite esencial se obturo mediante arrastre de vapor de agua, posteriormente se determinó el rendimiento de extracción, densidad relativa, índice de refracción y su solubilidad en etanol $(70 \% \mathrm{v} / \mathrm{v})$. La composición química fue evaluada mediante cromatografía de gases acoplada a espectrometría de masas (GC-MS). La actiridad antioxidante fue determinada mediante el método del radical libre 2,2-difenil1-picrilhidracilo (DPPH) y la capacidad de atrapamiento del catión radical ABTS*+ En los aceites esenciales de las especies Tagetes se logró identificar 26 componentes químicos para la especie Tagetes patula y 16 para Tagetes erecta., ambas especies presentaron como componentes principales a los monoterpenos $(61 \%)$ y sesquiterpenos (44\%). Los metabolitos bioactivos de aceites esenciales entre ambas especies de Tagetes fueron, $\beta$-trans-Ocimeno $(25,03 \%)$, Trans-Tagetona $(51,37 \%), \quad \beta$ Mirceno $(2,78)$ y $\beta$-Cariofileno $(1,17 \%)$. Los rendimientos de extracción oscilar on entre 0,05 y $0,048 \%$, la densidad entre 0,90 y $0,88(\mathrm{~g} / \mathrm{ml})$ con un índice de refracción de 1.493 y 1.482 y una solubilidad ( $\mathrm{v} / \mathrm{v})$ positiva entre ambas especies. La actividad antioxidante del aceite esencial en ambas especies mostró una variación entre 1,77 y $2,56 \mathrm{mg} / \mathrm{mL}$ para el DPPH y 21,02 a $41,06 \mathrm{mg} / \mathrm{mL}$ para BTS*+. Los Aceites esenciales de las especies Tagetes erecta y Tagetes patula son una fuente de metabolitos bioactivos fomentan potencialidades antimicrobianas y antioxidantes con fines de su uso como conservantes alimentarios.

Palabras claves: Cromatografia, densidad, aceite esencial, solubilidad.

\section{ABSTRACT}

The research aimed to investigate the bioactive metabolites and antioxidant activity of essential oils of two species of the genus Tagetes. The essential oil was obtained by means of water vapor drag, subsequently the extraction yield, relative density, refractive index and its solubility in ethanol $(70 \% \mathrm{v} / \mathrm{v})$ were determined The chemical composition was evaluated by gas chromatography coupled to mass spectrometry (GC-MS). The antioxidant activity was determined by the 2,2-diphenyl-1 picrylhydracil (DPPH) free radical method and the ABTS * + radical cation trapping capacity. In the essential oils of the Tagetes species, 26 chemical components were identified for the Tagetes patula species and 16 for Tagetes erecta. Both species had monoterpenes $(61 \%)$ and sesquiterpenes (44\%) as main components. The bioactive metabolites of essential oils between both Tagetes species were, $\beta$-transOcimeno (25.03\%), Trans-Tagetone (51.37\%), $\beta$-Myrcene (2.78) and $\beta$-Caryophyllene (1, $17 \%$ ). The extraction yields ranged between 0.05 and $0.048 \%$, the density between 0.90 and $0.88(\mathrm{~g} / \mathrm{ml})$ with a refractive index of 1.493 and 1.482 and a positive solubility ( $\mathrm{v} / \mathrm{v}$ ) between both species. The antioxidant activity of the essential oil in both species showed a variation between 1.77 and $2.56 \mathrm{mg} / \mathrm{mL}$ for DPPH and 21.02 to $41.06 \mathrm{mg} / \mathrm{mL}$ for BTS * +. The essential oils of the species Tagetes erecta and Tagetes patula are a source of bioactive metabolites that promote antimicrobial and antioxidant potentialities for the purpose of their use as food preservatives.

Keywords: Chromatography, density, essential oil, solubility.

\section{RESUMO}

A pesquisa tere como objetivo investigar os metabólitos bioativos e a atividade antioxidante de óleos essenciais de duas espécies do gênero Tagetes. O óleo essencial foi obtido por meio de arrasto de vapor d'água, posteriormente foram determinados o rendimento de extração, densidade relativa, indice de refração e sua solubilidade em etanol $(70 \% \mathrm{v} / \mathrm{v})$. A composição química foi avaliada por cromatografia gasosa acoplada a espectrometria de massas (GC-MS). A atividade antioxidante foi determinada pelo método do radical livre 2,2-difenil-1picrilidracila (DPPH) e pela capacidade de captura do cátion radical ABTS * +. Nos óleos essenciais das espécies Tagetes, foram identificados 26 componentes químicos para a espécie Tagetes patula e 16 para Tagetes erecta, sendo que ambas as espécies tinham monoterpenos $(61 \%)$ e sesquiterpenos $(44 \%)$ como componentes principais. Os metabólitos bioativos dos óleos essenciais entre as duas espécies de Tagetes foram, $\beta$-trans-Ocimeno $(25,03 \%)$, Trans-Tagetona $(51,37 \%), \quad \beta$ mirceno $(2,78)$ e $\beta$-cariofileno $(1,17 \%)$. Os rendimentos de extração variaram entre 0,05 e $0,048 \%$, a densidade entre 0,90 e $0,88(\mathrm{~g} / \mathrm{ml})$ com indice de refração de 1,493 e 1,482 e solubilidade positiva ( $\mathrm{v} / \mathrm{v}$ ) entre as duas espécies. A atividade antioxidante do óleo essencial em ambas as espécies apresentou variação entre 1,77 e $2,56 \mathrm{mg} / \mathrm{mL}$ para $\mathrm{DPPH}$ e 21,02 a 41,06 mg / mL para BTS * +. Os óleos essenciais das espécies Tagetes erecta e Tagetes patula são uma fonte de metabólitos bioativos que promovem potencialidades antimicrobianas e antioxidantes para uso como conservantes de alimentos.

Palavras-chave: Cromatografia, densidade, óleo essencial, solubilidade. 


\section{INTRODUCCIÓN}

Perú es uno de los 12 países con la mayor diversidad biológica, con aproximadamente $10 \%$ de flora mundial, estimada en 25000 especies, 30 de ellas son endémicas (Vasquez, et al., 2018). Existe un interés creciente en compuestos bioactivos y las propiedades antioxidantes de sustancias de fuentes naturales que potencialmente pueden ser utilizadas en las industrias alimentarias. Se sabe que los aceites esenciales de plantas aromáticas y medicinales poseen actividad biológica (Chrysargyris A., 2020; Tajkarimi, M. 2010). Los aceites esenciales son productos vegetales naturales que contiene una mezcla compleja por tanto tienen múltiples propiedades antimicrobianas (Ausama, A., Safar L., 2020). Siendo los constituyentes de los grupos de materias primas más importantes para la industria alimentaria, farmacéutica, de perfumería y afines (Olivera, et al., 2020). Tagetes es un género importante perteneciente a la familia Asteraceae (Marotti, M., Piccaglia, R. 2004), aromática, originaria de Centro y Sudamérica con una distribución cosmopolita debido a actividades antrópicas (Ojeda, et al. 2015). Tagetes patula, es cosechada en flor y se destila para extraer aceite esencial, éste es usado en perfumería; mezclado con aceite de sándalo produce el perfume attar genda. (Jacobs et al. 1994) presentando compuestos medicinales, antioxidantes, también como condimentos, ejecutando como tés y bebidas refrescantes. Es utilizado como repelente de insectos y hongos (Soule, 1993). Se cultiva en forma comercial para extraer un aceite esencial (aceite de Tagetes), así mismo es un sistema de monocultivo que está en función de los precios que compiten juntamente con la disposición de mano de obra (Torres, 2011). Tagetes erecta, también conocida como (cempasúchil) "flor del muerto", se caracteriza por su coloración y su contenido de carotenoides (Delgado et al. 2000). Las flores de Tagetes erecta son inflorescencias de tonos naranjas, amarillos y rojos. Las inflorescencias tienen capítulos con numerosas flores individuales (de 60 a 400). Estas flores pueden o no tener lígulas o pétalos (pétalas o apétalas). En el caso de T. patula, los capítulos tienen lígulas con el limbo elíptico de 8 a18 $\mathrm{mm}$ de largo y 6 a10 $\mathrm{mm}$ de ancho; las flores con forma de disco son de 12 a $40 \mathrm{~mm}$, y las tubulares de 10 a15 mm de largo (Gliman y Howe 1999, Villareal y Villaseñor 2004).

La gran importancia de Tagetes se debe a la presencia de aceite esencial en casi todas las partes de sus plantas, excepto en el tallo (Singh, V., Singh, B. 2003). Presenta 
actividades biológicas como actividades antibacterianas, antifúngicas, antivirales, antioxidantes, anticancerosas, acaricidas, nematicidas, insecticidas y alelopáticas (Gakuubi, M., Wanzala, W. 2016a). El creciente interés en las industrias de la alimentación, el sabor, la perfumería contribuye a la investigación de las condiciones ambientales afectan la composición cualitativa y el rendimiento (Swati, et al. 2020). A pesar de su importancia como especies alimentarias, las investigaciones en especies Tagetes erecta y Tagetes patula, con respecto a su composición química, diversidad genética y propiedades biológicas son limitadas. Por lo tanto, el objetivo fue determinar las propiedades físicas e identificar los metabolitos bioactivos y actividad antioxidante de los aceites esenciales de ambas especies del género Tagetes que crecen en forma silvestre y están adaptadas a ecosistemas de moderada altitud de la región andina del Perú.

\section{METODOLOGÍA}

\section{Extracción de aceites esenciales}

Para la extracción de los aceites esenciales se seleccionaron las hojas frescas de Tagetes erecta y Tagetes patula.; se utilizaron 2,5 kilogramos de hojas frescas de cada especie y se sometieron a extracción mediante destilación por arrastre a vapor de agua a una presión de 10 psi. Una vez destilado los aceites esenciales se separaron por diferencia de densidades utilizando un decantador florentino graduado. Luego se secaron sobre sulfato de sodio anhidro y se almacenaron a $4{ }^{\circ} \mathrm{C}$ hasta el momento de realizar análisis, los rendimientos de extracción se evaluaron según la ecuación 1.

$$
\% P=\frac{\text { Masa final de aceite esencial }(g)}{\text { !"\#" } \quad \& \text { \& \&+\&" } \quad)^{*} \text { muestra o }} \% \text { \%3(("4* }
$$

\section{Determinación de propiedades físicas del aceite esencial}

En los aceites esenciales obtenidos de cada especie se determinaron: la densidad relativa a $20^{\circ} \mathrm{C}$ de acuerdo a la norma técnica peruana: NTP 3129.081:1974; índice de refracción en refractómetro $\mathrm{ABBE}$; rotación óptica en polarímetro y solubilidad en etanol, para este último se empleó una solución al $70 \%$ tomando $100 \mu \mathrm{L}$ de aceite esencial. 
Determinación de compuestos químicos mediante cromatografía de gases acoplada a

\section{espectrometría de masas (GC-MS)}

El análisis de la composición química de los aceites esenciales fue identificado mediante Cromatografía de gases acoplada a espectrometría de masas (GC-MS) en el centro de investigación de productos naturales de la Universidad Peruano Cayetano Heredia.

\section{Evaluación de la actividad antioxidante de los aceites esenciales}

Para la determinación de la actividad antioxidante de los aceites esenciales de las especies del género Tagetes se emplearon dos metodologías:

\section{- Método del radical DPPH}

Se empleó el método de Brand-Williams et al., 1995 con algunas modificaciones, se prepararon diluciones en etanol acuoso de extractos hidroalcohólicos hasta obtener concentraciones de 0,0 a 150,0 $\mu \mathrm{g} / \mathrm{mL}$. Se combinaron $1,0 \mathrm{~mL}$ de cada dilución con 0,5 $\mathrm{mL}$ de una solución de $0,3 \mathrm{mM}$ de DPPH en etanol dejándose reaccionar a temperatura ambiente por 30 minutos, posteriormente se llevó a medir la absorbancia de las mezclas a $517 \mathrm{~nm}$ en el equipo de espectrofotometría.

\section{- Método del radical BTS*+}

Se empleó el método de Re et al., 1999 con algunas modificaciones. Se reacciono 3,5 $\mathrm{mM}$ de ABTS con 1,25 $\mathrm{mM}$ de persulfato de potasio. Las muestras se incubaron a temperaturas de $2-8{ }^{\circ} \mathrm{C}$ durante $16-24 \mathrm{~h}$. en oscuridad. El radical BTS*+ formado se diluyó con etanol hasta obtener una absorbancia de $0,7+-0,05$ a $734 \mathrm{~nm}$. A un volumen de $190 \mu \mathrm{L}$ de dilución del radical BTS*+ se agregó $10 \mu \mathrm{L}$ de la muestra de AE y se incubo a temperatura ambiente durante 5 minutos. Pasado el tiempo se llevó a determinar mediante el equipo espectrofotómetro a $734 \mathrm{~nm}$ en el lector de microplacas Themoscientific.

\section{Análisis estadístico}


Los análisis fueron realizados por triplicado, para la evaluación estadística se utilizó el diseño completamente al azar (DCA); el análisis de varianza se trabajó con 0,05 de significancia; al encontrar diferencia significativa se procedió a realizar la prueba de comparaciones de medias de Fisher (LSD) a un nivel de $\alpha=0,05$. Los datos fueron procesados con la ayuda de los programas estadísticos Centurión XVII y la hoja de cálculo Microsoft Excel 2019.

\section{RESULTADOS Y DISCUSIÓN}

\section{Rendimiento y propiedades físicas de los aceites esenciales}

La determinación de las propiedades físico-químicas nos permiten conocer la calidad y el control de pureza en los aceites esenciales. De acuerdo a la tabla 1 se muestra el porcentaje de rendimiento de extracción y las propiedades físicas de aceites esenciales de ambas especies del género Tagetes.

\section{Tabla 1.}

Rendimiento y propiedades fisicas de los aceites esenciales de Tagetes erecta y Tagetes patula.

\begin{tabular}{lll}
\hline Análisis & Tagetes erecta & Tagetes patula \\
\hline Rendimiento & $0,05 \pm 0,002^{\mathrm{a}}$ & $0,048 \pm 0,001^{\mathrm{a}}$ \\
Densidad $(\mathrm{g} / \mathrm{ml})$ a $24^{\circ} \mathrm{C}$ & $0,900 \pm 0,0004^{\mathrm{a}}$ & $0,882 \pm 0,0043^{\mathrm{b}}$ \\
Índice de refracción a $24^{\circ} \mathrm{C}$ & $1,493 \pm 0,05^{\mathrm{a}}$ & $1,482 \pm 0,04^{\mathrm{a}}$ \\
Solubilidad EtOH $70 \%(\mathrm{v} / \mathrm{v})$ & Positiva & Positiva \\
Gravedad específica a $20^{\circ} \mathrm{C}$ & $0,872 \pm 0,01^{\mathrm{a}}$ & $0,945 \pm 0,034^{\mathrm{b}}$
\end{tabular}

Los aceites esenciales de Tagetes erecta. y Tagetes patula, no presentaron diferencias significativas en el porcentaje de rendimiento. El rendimiento del aceite esencial depende de la planta y el distrito donde se cultiva (Preedy, V. 2016). De acuerdo a los resultados de propiedades físicas del aceite esencial, la densidad mostro una variación para ambas especies del género Tagetes, sin embargo, el índice de refracción no mostro una variación entre ambas especies. La presencia de un valor menor del índice de refracción y densidad está relacionada con una cantidad de fenoles (Husnu, K. y Baser, C., 2007). El índice de refracción de ambas especies presentó valores elevados, los valores elevados indican presencia de compuestos de alto peso molecular tales como sesquiterpenos y diterpenos y eventualmente oleorresinas en concentraciones altas 
(Simard, S. y Hachey, J. 1988) así mismo, siendo un indicativo de aceites esenciales de mayor calidad y pureza. De acuerdo a los resultados de gravedad especifica de los aceites esenciales de ambas especies se encontró diferencias significativas con presencia a mayor calidad $(0,945 \pm 0,034)$ en el aceite esencial de Tagetes patula, encontrándose valores obtenidos similares según reportes en estudios (Chalchat, J. y Garry, R., 1995).

\section{Composición química de los aceites esenciales de dos especies del género Tagetes}

Los componentes mayoritarios de los aceites esenciales de ambas especies del género Tagetes se muestra en la tabla 2.

\section{Tabla 2}

Componentes mayoritarios detectados en los aceites esenciales de Tagetes erecta y Tagetes patula.

$$
\% \text { de abundancia relativa, (TR\%) }
$$

\section{Compuesto}

$$
\text { Tagetes erecta }
$$

Tagetes patula

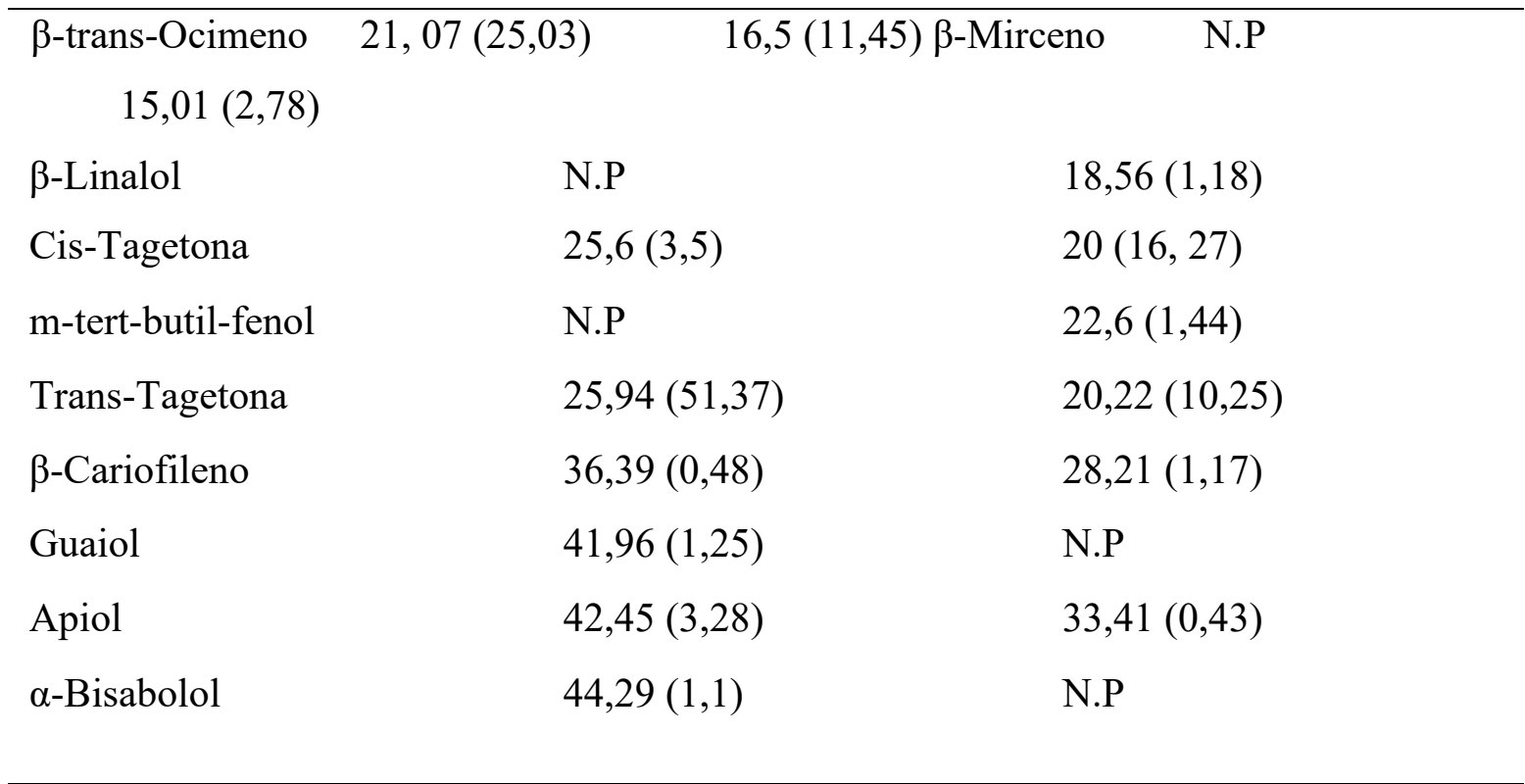

Tiempo de retención (TR) y abundancia relativa (\%) de los aceites esenciales, No presentó (NP). En el análisis de la composición química se detectaron y cuantificaron un total 26 compuestos químicos en el aceite esencial de Tagetes erecta con fracción 
principal en monoterpenos en $(61,00 \%)$ y 16 compuestos químicos para el aceite esencial de Tagetes patula encontrándose como fracción principal a los monoterpenos (50,0\%), entre ambas especies se obtuvo una desviación estándar por debajo del $5 \%$ entre los porcentajes de cada analito en ambas columnas utilizadas. Se identificaron como metabolitos bioactivos en los aceites esenciales en las especies del género Tagetes al $\beta$-trans-Ocimeno, Trans-Tagetona, Cis-Tagetona, $\beta$-Mirceno y $\beta$ Cariofileno. El análisis de los componentes químicos en los aceites esenciales de las especies Tagetes erecta y Tagetes patula, mostraron mayormente la presencia de los siguientes compuestos: Trans-Tagetona, $\beta$-trans-Ocimeno, Cis-Tagetona, $\beta$ Cariofileno y Apiol. Los aceites esenciales de la especie Tagetes spp. son ricos en hidrucarburos monoterpenicos (Ocimenos, limoneno, terpineno, mirceno y cetonas monoterpenicas aciclicas (tagetona, dihidrotagetona, y tagetenona) que son los odorates primarios además de cantidades menores de hidrocarburos sesquiterpenicos compuestos oxigenados (Salehi, et al., 2018). En cuanto a sus bioactividades de la familia de especies del género Tagetes se encontró una actividad antibacteriana de fuerte a leve contra cepas de bacterias gran-positivas y gran-negativas probadas en el estudio (Asuma, A. Safar, L. 2020) Actividad antioxidante de AE de Tagetes erecta y Tagetes patula

En la tabla 3. Se muestra los resultados de actividad antioxidante en los aceites esenciales de ambas especies del género Tagetes, mediante los métodos de DPPH y ABTS

\section{Tabla 3}

Actividad antioxidante mediante métodos DPPH Y ABTS.

Aceite esencial

\section{Métodos}

\section{$\operatorname{DPPH~IC}_{50}(\mathrm{mg} / \mathrm{mL}) \quad \operatorname{ABTS~IC}_{50}(\mathrm{mg} / \mathrm{mL})$}

\begin{tabular}{lll}
\hline Tagetes erecta & $1,77 \pm 0,02$ & $21,02 \pm 0,14$ \\
Tagetes patula & $2,56 \pm 0,12$ & $41,06 \pm 0,23$
\end{tabular}

Se encontró diferencias significativas en la actividad antioxidante de ambas muestras de Tagetes como se muestra en la tabla 3. De acuerdo a la metodología de DPPH el $\mathrm{IC}_{50}$ varió de $1,77 \mathrm{mg} / \mathrm{mL}$ a $2,56 \mathrm{mg} / \mathrm{mL}$, sin embargo, el $\mathrm{IC}_{50}$ de BTS*+ vario de 21,02 a 41,06 mg/mL, encontrándose una actividad antioxidante mas alto el valor de $\mathrm{IC}_{50} 41,06 \mathrm{mg} / \mathrm{mL}$. El aceite esencial de Tagetes erecta presento una $\mathrm{IC}_{50}$ más bajo 
$1,77 \mathrm{mg} / \mathrm{mL}$, respectivamente exhibió una considerable actividad de eliminación de radicales DPPH en comparación con el método de BTS*+. La diferencia en la actividad antioxidante entre ambas muestras podría atribuirse a la presencia de los monoterpenos en sus compuestos polifenólicos, los monoterpenos oxigenados conducen a actividades antioxidantes, antibacterianas y antifúngicas más altas (Rostaei, M. 2018b; Deba, F. 2008; Tohidi, B. 2017).

\section{CONCLUSIONES}

En el estudio se logró determinar los metabolitos bioactivos de los aceites esenciales de las especies de Tagetes erecta y Tagetes patula, encontrándose en mayor en abundancia a los metabolitos bioactivos: $\beta$-trans-Ocimeno, Trans-Tagetona, CisTagetona, $\beta$-Mirceno y $\beta$ Cariofileno, siendo los monoterpenicas acíclicas, con efectos significativos como antioxidantes, inhibidores de enzimas, precursores de sustancias tóxicas y pigmentos beneficiosos para alcanzar su potencial en biotecnología. La abundancia en monoterpenos conducen a actividades antioxidantes, encontrándose en el estudio mayor presencia de antioxidantes en la especie de Tagetes patula. Las propiedades físicas de ambas especies del género Tagetes se encontró en los rangos de calidad para aceites esenciales.

\section{REFERENCIAS BIBLIOGRÁFICAS}

Ausama A Safar 1, Anwar O Ghafoor 2, Dara Dastan. (2020). Composición química, actividades antibacterianas y antioxidantes del aceite esencial de Tagetes patula L. criado en Erbil, Iraq. Departamento de producción vegetal, Instituto Técnico Khabat, Universidad Politécnica de Erbil, Erbil, Región del Kurdistán, Irak; Departamento de Biología, Facultad de Educación, Universidad SalahaddinErbil, Erbil, Región del Kurdistán, Irak 2 Departamento de Biología, Facultad de Educación, Universidad Salahaddin-Erbil, Erbil, Región del Kurdistán, Irak.

Brand-Williams, W., M.E. Cuvelier \& C. Berset, (1995). Use of a free radical method to evaluate antioxidant activity, LebensmittelWissenchaft und Technologie: 28(1), 25-30.

Chalchat, J.C.; Garry, R.P.; Muhayimana, A. (1995). Essential oil of Tagetes minuta from Rwanda and France: Chemical composition according to harvesting 
location, growth stage and part of plant extracted. J. Essent. Oil Res. 7(4): 375386.

Chrysargyris A, Mikallou M, Petropoulos S, Tzortzakis N. (2020). Perfilado de componentes de aceites esenciales y polifenoles para determinar su actividad antioxidante de plantas medicinales y aromáticas cultivadas en diferentes condiciones ambientales. Agronomía; 10 (5): 727.

Deba, F., Xuan, T.D., Yasuda, M. \&Tawata, S. (2008). Chemical composition and antioxidant, antibacterial and antifungal activities of the essential oils from Bidens pilosa linn. Var. Radiata. Food Control 19, 346-352.

DelgadoVargas, F., A.R. Jimenez \& O. Paredes-Lopez. 2000. Natural Pigments: Carotenoids, Anthocyanins, and Betalains Characteristics, Biosynthesis, Processing, and Stability. Critical Reviews in Food Science and Nutrition 40(3): 173-289.

G. Oliveira-Everton, R.J. Pereira-Araújo, A.B. da Silva dos Santos, P.V. Serra-Rosa, R.G. de OliveiraCarvalho Junior, A.M. Teles, P.R. Barros-Gomes, V.E. Mouchrek Filho, (2020). Caracterização química, atividade antimicrobiana e toxicidade dos óleos essenciais da Pimenta dioica L. (pimenta da Jamaica) e Citrus sinensis L. Osbeck (laranja doce), Rev. Colomb. Cienc. Quím. Farm., $49(3), 641655$

Gakuubi, M.M., Wanzala, W., Wagacha, J.M., Dossaji, S.F. (2016a). Bioactive properties of Tagetes minuta L. (Asteraceae) essential oils: a review. Amer. J. Essent. Oil Nat. Prod. 4, 27-36.

GLIMAN, E.F. \& T. HOWE. 1999. Tagetes erecta. Institute of Food and Agricultural Sciences. Fact Sheet FPS-569: 1-3.

Hüsnü, K., Başer, C., y Demirci, F. (2007). Chemistry of Essential Oils. Flavours and Fragrances, (1), 43-86.

Jacobs, J. J.; A. Engelberts; A. F. Croes; G. J. Wullems. (1994). "Thiophene synthesis and distribution in young developing plants of Tagetes patula and Tagetes erecta"; Journal of Experimental Botany 45: 14591466 
Marotti M, Piccaglia R, Biavati B, Marotti I. (2004). Caracterización y evaluación del rendimiento de aceites esenciales de diferentes especies de Tagetes. J Essent Oil Res; 16: 440-4.

Ojeda, M.S., Torkel Karlin, U.O., Martinez, G.J., Massuh, Y., Ocaño, S.F., Torres, L.E., Chavez, A.G. (2015). Arizio, O., Curioni, A. Planta Aromáticas y Medicinales Modelos para su Domesticación, Producción y Usos Sustentables [Aromatic and medicinal plant models for domestication, production and sustainable uses]. Ed. UNC, Córdoba, Argentina.

Preedy, V. 2016. Essential oils in food presservation, flavor and safety. Academic Press is an imprint of Elsevier, Amsterdam. 932 pp.

Rostaei, M., Fallah, S., Lorigooini, Z., Abbasi Surki, A. (2018b). Crop productivity and chemical compositions of black cumin essential oil in sole crop and intercropped with soybean under contrasting fertilization. Ind. Crop Prod. 125, 622-629. https://doi. org/10.1016/j.indcrop. 09.044.

Salehi B, Valussi M, Morais-Braga MFB, Carneiro JNP, Leal A, Coutinho HDM, et al. (2018). Tagetes spp. Aceites esenciales y otros extractos: Caracterización química y actividad biológica. Moléculas. 23: 2847.

Simard, S.; Hachey, J.M.; Collin, G.J. (1988). The variations of essential oil composition during the extraction process. The case of Thuja occidentalis L. and Abies balsamea (L.) Mill. J. Wood Chem. Technol. 8: 561-573.

Singh, V., Singh, B., Kaul, V.K. (2003). Domestication of wild marigold (Tagetes minuta L.) as a potential economic crop in western Himalaya and north Indian plains. Econ. Bot. 57, 535-544.

Swati, W., Srijana M., Vinod, B., Rakshak K., Rakesh K. (2020). Variability in chemical composition and antimicrobial activity of Tagetes minuta L. essential oil collected from different locations of Himalaya. Academy of Scientific and Innovative Research, New Delhi, India.

Tajkarimi, M.; Ibrahim, S. \& Cliver, D. (2010). Hierbas antimicrobianas y compuestos de especias en los alimentos. Control de alimentos. 21, 1199-1218, 
Tohidi, B., Rahimmalek, M., Arzani, A. (2017). Essential oil composition, total phenolic, flavonoid contents, and antioxidant activity of Thymus species collected from different regions of Iran. Food Chem. 220, 153-161.

Torres, S. (2011). Marigold o cempasúchil, la flor maravilla: Escasea en el mundo. Article appeared in Industria Avícula, guadlajara, Jalisco, México.

Vásquez Ocmín, P.; Cojean, S.; Rengifo, E.; Suyyagh-Albouz, S.; Amasifuen Guerra, C.A.; Pomel, S.; Cabanillas, B.; Mejía, K.; Loiseau, P.M.; Figadère, B.; Maciuk, A. (2018). Antiprotozoal activity of medicinal plants used by IquitosNauta road communities in Loreto (Peru). 210: 372-385

Villareal, J.A. \& J.L. Villaseñor. 2004. Flora de Veracruz: Compositae y tribu Tagetae. Instituto de Ecología y University of California 135: 1-67. 